\title{
MORFOMETRIA DE ANASTREPHA FRATERCULUS (WIED) (DIPTERA: TEPHRITIDAE) RELACIONADA A HOSPEDEIROS NATIVOS, MYRTACEAE
}

\author{
D.R.G. Pereira-Rêgo, S.M. Jahnke, L.R. Redaelli, N. Schaffer
}

Universidade Federal do Rio Grande do Sul, Faculdade de Agronomia, Departamento de Fitossanidade, Av. Bento Gonçalves, 1277, CEP 91540-000, Porto Alegre, RS, Brasil. Email: dro.dro@hotmail.com

\section{RESUMO}

A mosca-das-frutas, Anastrepha fraterculus, tem, como principais hospedeiros primários, frutos de Myrtaceae. $\mathrm{O}$ tamanho do inseto pode ser indicador de aptidão e fecundidade, sendo os estudos morfométricos utilizados para auxiliar nestas identificações. Este trabalho objetivou verificar a associação entre peso dos frutos, pupários e tamanho das asas das moscas-das-frutas que se desenvolveram em diferentes mirtáceas. Foram amostrados frutos de araçazeiro-amarelo, araçazeiro-vermelho, goiabeira e goiabeira-serrana em cinco árvores de cada espécie. Os frutos foram pesados e os pupários oriundos destes pesados e individualizados. Registrou-se o número, sexo e área total da asa direita das moscas. Comparou-se o peso médio dos frutos, dos pupários e o tamanho de asas. O peso médio dos pupários foi maior em goiaba e araçá-vermelho. A maior área média de asa foi registrada nos indivíduos emergidos de goiabas e a menor, nos de araçá-amarelo. O peso dos frutos e dos pupários em araçá-amarelo, vermelho e goiaba-serrana não apresentaram associação significativa. Nas goiabeiras, a correlação entre o peso dos frutos e o dos pupários foi negativa. O peso dos frutos e a área da asa das moscas provenientes das espécies de mirtáceas não evidenciaram correlação. Os resultados indicam que a mosca é capaz de desenvolver-se de forma semelhante nas espécies de mirtáceas amostradas.

PALAVRAS-CHAVE: Morfometria, Mosca-das-frutas, Myrtaceae.

\section{ABSTRACT}

MORPHOMETRY OF ANASTREPHA FRATERCULUS (WIED) (DIPTERA: TEPHRITIDAE) RELATED TO NATIVE MYRTACEAE HOSTS. The fruit-fly Anastrepha fraterculus uses the myrtacean fruits as its main primary hosts. The insect size might be an indicator of aptitude and fecundity, and morphometric studies are used to help in those identifications. This study aimed to verify the association among fruit weight, puparial weight and wing size of fruit-flies that developed in different myrtacean species. Yellow strawberry guava, cattle guava, guava and feijoa fruits were sampled from 5 trees of each species. The fruits were weighed, and the puparia originated from them were also weighed and individualized. The number, gender and total right-wing area of the flies were registered. Mean fruit and puparial weights and wing sizes were compared. Mean puparial weight was higher in guava and cattle guava. The largest mean wing area was recorded for individuals from guavas; the smallest, for those from yellow strawberry guava. The fruit and puparial weights did not present a significant association in yellow strawberry guava, cattle guava and feijoa. In guavas, the correlation between fruit and puparial weights was negative. The fruit weight and wing area in all myrtacean species did not show any correlation. These results indicate the fly is able to develop similarly in all sampled myrtacean species.

KEY WORDS: Morphometric, fruit-flies, Myrtaceae.

\section{INTRODUÇÃO}

A fruticultura no Brasil assume cada vez mais uma posição importante no setor de produção primária e, no Rio Grande do Sul, tem se tornado uma significativa alternativa econômica (Joño, 2004).

Uma das principais pragas da fruticultura brasileira é a mosca-das-frutas sul-americana, Anastrepha fraterculus (Wied.) (Diptera: Tephritidae). Segundo Zucchi (2008), no Brasil são conhecidas 102 espécies desse gênero. No Rio Grande do Sul, A. fraterculus é a espécie mais abundante, ocorrendo em diversas frutíferas (KovALESKI et al., 2000). Associadas a A. fraterculus são registradas 76 espécies de plantas, sendo que Myrtaceae destaca-se como uma das principais famílias com hospedeiros primários da espécie (Malavasi et al., 2000; Zucchi, 2008). 
A morfometria é a formalização matemática das diferenças entre as formas (SILVA, 2008). Na entomologia, o estudo morfométrico aplica-se como ferramenta que auxilia os diversos ramos desta ciência (PIRES, 2006). CruZ et al. (2000), trabalhando com pupários de moscas-das-frutas, referiram o peso destes como um fator importante para processos fisiológicos relacionados à formação do adulto. Desta forma, o tamanho pode ser um bom indicador de aptidão ou de fecundidade.

Dentre as estruturas corporais utilizadas para estudos morfométricos busca-se utilizar estruturas que possibilitem medidas mais acuradas sem possibilidade de distorções. Para tanto as asas se justificam, por serem planas e de fácil manuseio. (VAz et al., 2004).

Neste sentido, os objetivos deste trabalho foram verificar a associação entre peso dos frutos coletados na copa e no solo e em diferentes estágios de maturação na mesma frutífera, o peso dos pupários e o tamanho das asas de indivíduos de A. fraterculus; e comparar a média destas medidas entre quatro espécies de mirtáceas no Rio Grande do Sul.

\section{MATERIAL E MÉTODOS}

As amostragens foram realizadas de janeiro a março de 2009 em cinco árvores de cada uma das seguintes espécies: araçá-amarelo (Psidium cattleyanum Sabine var. Lucidum), araçá-vermelho (Psidium cattleyanum Sabine), em janeiro e fevereiro; goiaba (Psidium guajava L. var. Paluma) em fevereiro e março e goiaba-serrana (Acca sellowiana Berg.), em março.

As goiabas foram obtidas em um pomar experimental, localizado no Centro Agrícola Demonstrativo da Prefeitura Municipal de Porto Alegre (CAD), $\left(30^{\circ} 07^{\prime} \mathrm{S}, 51^{\circ} 05^{\prime} \mathrm{O}\right)$, Porto Alegre, RS. Os araçás-amarelos foram coletados em árvores de três locais, uma no CAD, três no Campus do Vale da UFRGS $\left(30^{\circ} 07^{\prime} \mathrm{S} 51^{\circ} 05^{\prime} \mathrm{O}\right)$, Porto Alegre, RS, e uma na Estação Experimental da Embrapa Uva e Vinho, em Bento Gonçalves, RS (29 $\left.09^{\prime} \mathrm{S} 51^{\circ} 31^{\prime} \mathrm{O}\right)$. Os araçás-vermelhos foram coletados de três árvores, uma no Campus do Vale da UFRGS, uma na Estação Experimental da Embrapa Uva e Vinho e uma em propriedade particular em Montenegro, RS (29 $40^{\circ}$ S, $51^{\circ} 32^{\prime} \mathrm{O}$ ). A coleta de goiabas-serranas foi realizada num pomar com manejo orgânico, situado na mesma propriedade particular em Montenegro, RS. Todas as árvores de onde foram coletados os frutos não recebiam tratamentos fitossanitários.

As coletas preconizaram frutos íntegros de dois locais: copa e solo (sob a copa da árvore). Nas coletas efetuadas nas copas dos araçazeiros e das goiabeiras, foram amostrados frutos de três estágios de maturação: verde, intermediário (semimaduro) e maduro, determinados visualmente através da cor. Nos araçazeiros-amarelo evermelho, em cada estágio de maturação e no solo, foram coletados 20 frutos, totalizando 80 por árvore. Nas goiabeiras, no solo e de cada estágio de maturação, amostraram-se dez frutos, totalizando 40 por árvore. Nas goiabeirasserranas coletou-se somente 20 frutos da copa e 20 do solo, totalizando 40 frutos por árvore, sem discriminar os estágios de maturação, uma vez que nesta frutífera não é possível diferenciá-los, visualmente, com clareza.

Os frutos coletados foram levados para laboratório em sacos plásticos acondicionados em caixas de isopor. No laboratório estes foram lavados com água e hipoclorito $0,03 \%$ e pesados em balança de precisão marca Gehaka, modelo BG 200. Posteriormente, foram depositados, individualmente, em recipientes plásticos de $500 \mathrm{~mL}$ contendo, no fundo, uma camada ( $\pm 5 \mathrm{~cm}$ de espessura) de areia esterilizada. Os copos foram cobertos com tecido tipo "voil" e dispostos em uma casa de vegetação sob condições ambientais até a formação de pupários. Cada recipiente foi numerado, registrando-se a data e o local de coleta, o ponto de maturação do fruto e o local na árvore (copa ou solo).

Para a obtenção dos pupários, a areia depositada no fundo dos recipientes foi peneirada aos 12 dias após a coleta. Os pupários foram individualizados e acondicionados em potes plásticos de $100 \mathrm{~mL}$ contendo uma camada ( $\pm 1 \mathrm{~cm}$ de espessura) de areia esterilizada tratada com nipagin $(0,01 \%)$ e um frasco, tipo Ependorf, contendo água destilada, tampado com um chumaço de algodão hidrofílico, para manutenção da umidade, e armazenados em câmara a $25 \pm 2^{\circ} \mathrm{C}, 60 \pm 10 \%$ UR e fotoperíodo 16L:8E, até a emergência. Cinco pupários, com idade em torno de 12 dias, de cada local de coleta e ponto de maturação, foram escolhidos aleatoriamente e pesados. Os recipientes foram revisados a cada dois dias durante os meses de fevereiro, março, abril e maio de 2009 para a retirada dos adultos que foram sexados e conservados em álcool 70\%.

Foram feitos registros do número e do peso dos frutos, número total de pupários e o peso dos selecionados e número e sexo das moscas emergidas. Foi medida a área total da asa direita (VAz et al., 2004) das moscas. Para tanto, utilizou-se o software UTHSCSA Image Tool, que usa como base imagens digitais para obter os valores lineares e deárea destas. As imagens foram obtidas com a câmera fotográfica marca Nikon Coolpix 4.500 acoplada a um estereomicroscópio.

Foram pesados 400 frutos (100 de cada espécie de mirtácea) e um pupário oriundo de cada um destes totalizando 400 pupários. Obteve-se 318 moscas-dasfrutas, das quais a área da asa direita foi medida, os demais pupários foram desconsiderados, por serem inviáveis ou por terem originado parasitoides. 
Comparou-se os pesos médios dos pupários e tamanhos de asas de indivíduos oriundos dos diferentes frutos e pontos de maturação. As comparações foram feitas entre as espécies de frutíferas, desconsiderando-se o local de coleta e o estagio de maturação (números totais) e, intraespécies, comparando-se frutos da copa e solo e os diferentes estágios de maturação.

Para estas comparações utilizou-se análise de variância (Anova 5\%) e a normalidade dos dados foi testada pelo teste de D'Agostino. Foram realizados testes de correlação e regressão linear simples para avaliar a relação e a influência do peso dos frutos no peso dos pupários e, destes dois, no tamanho da área da asa das moscas, através dos softwares Microsoft Excel e Bioestat 5.0.

\section{RESULTADOS}

Todas as moscas-das-frutas foram identificadas como A. fraterculus, conforme chave proposta por ZuCCHI (2000b).

Comparando os valores médios totais entre as frutíferas, o peso médio dos pupários oriundos da goiaba $(16,6 \pm 0,60 \mathrm{mg})$ diferiu das demais espécies com exceção do araçá-vermelho $(14,5 \pm 0,68 \mathrm{mg})$ (Fig. 1A). Os pupários oriundos do araçá-amarelo $(13,4 \pm 0,64 \mathrm{mg})$ não diferiram apenas dos da goiaba- serrana (11,5 \pm 0,59 mg) e do araçá-vermelho (Fig. 1A).

Em relação àárea média da asa direita das moscasdas-frutas, as que se desenvolveram em goiaba (12,9 $\left.\pm 0,20 \mathrm{~mm}^{2}\right)$, araçá-vermelho $\left(12,6 \pm 0,22 \mathrm{~mm}^{2}\right)$ e goiaba-serrana $\left(12,2 \pm 0,28 \mathrm{~mm}^{2}\right)$ foram semelhantes, não apresentando diferença estatística entre suas médias. As oriundas do araçá-amarelo $(12,0 \pm 0,23$ $\left.\mathrm{mm}^{2}\right)$ foram significativamente menores do que as de goiaba não se diferenciando das demais (Fig. 1B).

Os araçás-amarelos no estágio verde pesaram, em média, 9,6 $60,75 \mathrm{~g}$, os semimaduros $8,9 \pm 0,72$ g e os maduros $7,9 \pm 0,66$ g e não diferiram entre si $(\mathrm{H}=2,6003 ; \mathrm{gl}=2 ; \mathrm{P}=0,2725)$. Considerando-se todos os frutos obtidos da copa, o peso médio foi de $8,8 \pm 0,41 \mathrm{~g}$ e, nos coletados no solo, sob a copa das árvores, foi de $8,3 \pm 0,70 \mathrm{~g}$, semelhantes entre si ( $\mathrm{H}$ $=0,4332 ; \mathrm{gl}=1 ; \mathrm{P}=0,5104)$ (Tabela 1).

Nos frutos verdes de araçá-amarelo, registrouse uma correlação positiva entre o peso dos frutos e o dos pupários $\left(\mathrm{r}=0,637, \mathrm{p}=0,001 ; \mathrm{R}^{2}=0,3\right.$, $\mathrm{P}=0,007)$, sendo que $30 \%$ da variação do peso dos pupários pode ser explicada pelo peso dos frutos. Quando analisados os frutos coletados em diferentes locais (copa e solo), não foi apurada associação com qualquer parâmetro comparado. A pequena variação no tamanho dos frutos, dessa espécie de frutífera, pode explicar a ausência de associação entre o tamanho dos indivíduos e o peso dos frutos.

Tabela 1 - Médias ( \pm EP) do peso de frutos (g) e pupários (mg) e da área da asa direita de Anastrepha fraterculus (mm²) oriundas de araçás-amarelos (Psidium cattleyanum var. Lucidum), coletados em Bento Gonçalves e Porto Alegre, de janeiro a fevereiro de 2009.

\begin{tabular}{lccc}
\hline \multirow{2}{*}{ Estágio de maturação/local } & \multicolumn{2}{c}{ Peso } & \multirow{2}{*}{ Área da asa $\left(\mathrm{mm}^{2}\right)$} \\
\cline { 2 - 3 } & frutos $(\mathrm{g})$ & pupários $(\mathrm{mg})$ & $12,2 \pm 0,54$ \\
\hline Verde & $9,6 \pm 0,75$ & $12,7 \pm 1,20$ & $12,1 \pm 0,40$ \\
Semimaduro & $8,9 \pm 0,72$ & $12,8 \pm 1,72$ & $12,1 \pm 0,45$ \\
Maduro & $7,9 \pm 0,66$ & $12,9 \pm 1,12$ & $12,1 \pm 0,26$ \\
Copa (total) & $8,8 \pm 0,10$ & $12,8 \pm 0,76$ & $11,7 \pm 0,50$ \\
Solo & $8,2 \pm 0,70$ & $15,1 \pm 1,16$ & $12,0 \pm 0,23$ \\
\hline Total & $8,6 \pm 0,35$ & $13,3 \pm 0,64$ & \\
\hline
\end{tabular}

Médias não diferiram estatisticamente (Kruskal Wallis, $\mathrm{P}>0,05$ ).
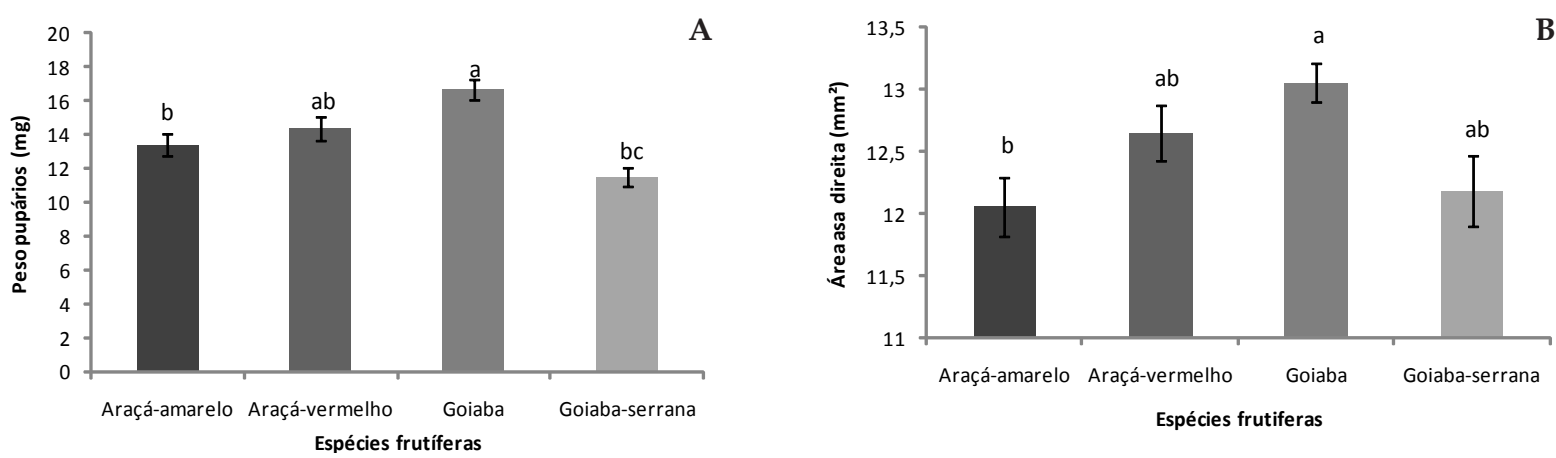

Fig. 1 - (A) Média ( \pm desvio-padrão) do peso de pupários (mg) de Anastrepha fraterculus para cada espécie de frutífera amostrada; (B) Média ( \pm desvio-padrão) da área da asa direita de Anastrepha fraterculus ( $\mathrm{mm}^{2}$ ) para cada espécie de frutífera amostrada (barras com mesma letra não diferem entre si pelo teste de Kruskal Wallis, a 5\% de probabilidade). 
Os araçás-vermelhos pesaram, em média, 4,9 $0,60 \mathrm{~g}$ no estágio verde, $5,1 \pm 0,55 \mathrm{~g}$ no semimaduro e 6, $5 \pm 0,75 \mathrm{~g}$ no maduro, não diferindo $(\mathrm{H}=2,2589$; $\mathrm{gl}=2 ; \mathrm{P}=0,3232)$ entre si. Considerando a totalidade dos frutos coletados na copa, dos três estágios, a média foi de $5,5 \pm 0,37 \mathrm{~g}$, não diferindo dos coletados no solo $5,5 \pm 0,70 \mathrm{~g}(\mathrm{H}=0,1341 ; \mathrm{gl}=1 ; \mathrm{P}=0,7142)$ (Tabela 2).

O peso médio dos pupários, assim como a área média das asas das moscas-das-frutas, oriundas de araçás-vermelhos, nos três estágios de maturação, não diferiram entre si. O mesmo foi observado quando estes parâmetros foram comparados entre os frutos da copa e do solo (Tabela 2).

No solo, registrou-se uma correlação positiva $\left(r=0,436, p=0,042 ; R^{2}=0,203, p=0,030\right)$ entre o peso dos pupários e o peso dos frutos, embora somente $30 \%$ da variação dos pupários esteja relacionada aos frutos. Apesar do peso médio dos pupários não diferir entre os estágios de maturação, constatou-se uma correlação positiva entre o peso dos frutos e dos pupários $\left(\mathrm{r}=0,487, \mathrm{p}=0,019 ; \mathrm{R}^{2}=0,0179, \mathrm{p}=0,042\right)$, nos maduros, sendo que $17,9 \%$ da variação do peso dos pupários foi explicada pelo peso dos frutos.

Os frutos verdes de goiaba pesaram, em média, $61,1 \pm 5,04 \mathrm{~g}$ e foram significativamente mais leves que os semimaduros $(92,8 \pm 9,38 \mathrm{~g})$, e maduros $(79,2$ $\pm 6,14 \mathrm{~g})(\mathrm{H}=12,2652 ; \mathrm{gl}=2 ; \mathrm{P}=0,0022)$ (Tabela $3)$. Não houve diferença entre o peso dos frutos coletados na copa $(77,4 \pm 4,34 \mathrm{~g})$ e no solo $(74,2 \pm$ $8,36 \mathrm{~g})(\mathrm{F}=0,131 ; \mathrm{gl}=1 ; \mathrm{P}=0,7191)$. O peso médio dos pupários não diferiu, nem entre os estágios de maturação, nem em relação ao local coletado, assim como a área média das asas das moscas (Tabela 3).

Em goiaba, registrou-se uma associação negativa entre o peso dos frutos e o dos pupários, em frutos maduros $\left(\mathrm{r}=-0,509, \mathrm{p}=0,016 ; \mathrm{R}^{2}=0,280, \mathrm{p}=0,011\right)$, considerando toda a copa $\left(r=-0,284, p=0,017 ; R^{2}=\right.$ $0,056, p=0,044)$ e os frutos da copa e do solo juntos ( $\mathrm{r}$ $\left.=-0,237, p=0,021 ; R^{2}=0,229, p=0,024\right)$. Verificou-se em todas as associações que, quanto maior o peso dos frutos, menor o peso dos pupários.

Na goiaba-serrana o peso médio dos frutos coletados na copa $(14,2 \pm 1,52 \mathrm{~g})$ foi significativamente menor do que os do solo, (17,6 $6 \pm 1,13 \mathrm{~g}$, $)(\mathrm{H}=6,1577$; $\mathrm{gl}=1 ; \mathrm{P}=0,0131$ ). Considerando toda a planta (copa + solo), o peso médio dos frutos foi de 15,9 \pm 0,97 g. O peso médio dos pupários e a área média das asas das moscas não diferiram estatisticamente em relação ao local de coleta (Tabela 4).

Registrou-se, em goiaba-serrana, uma associação entre o peso dos frutos coletados no solo e o peso dos pupários $\left(\mathrm{r}=-0,472, \mathrm{p}=0,031 ; \mathrm{R}^{2}=0,195, \mathrm{p}=0,040\right)$, sendo este influenciado pelo primeiro.

Tabela 2 - Médias ( \pm EP) do peso de frutos (g) e pupários (mg) e da área da asa direita de Anastrepha fraterculus (mm²) oriundas de araçás-vermelhos (Psidium cattleyanum), coletados em Bento Gonçalves, Montenegro e Porto Alegre, RS, de janeiro a fevereiro de 2009.

\begin{tabular}{lccc}
\hline \multirow{2}{*}{ Estágio de maturação/local } & \multicolumn{3}{c}{ Peso } \\
\cline { 2 - 3 } Área da asa $\left(\mathrm{mm}^{2}\right)$ \\
\hline Verde & frutos $(\mathrm{g})$ & pupários $(\mathrm{mg})$ & $12,4 \pm 0,48$ \\
Semimaduro & $4,9 \pm 0,60$ & $18,4 \pm 6,24$ & $12,1 \pm 0,57$ \\
Maduro & $5,0 \pm 0,55$ & $16,0 \pm 1,37$ & $12,8 \pm 0,38$ \\
Copa (total) & $6,4 \pm 0,75$ & $15,5 \pm 1,29$ & $12,4 \pm 0,27$ \\
Solo & $5,4 \pm 0,37$ & $16,6 \pm 2,00$ & $13,1 \pm 0,28$ \\
\hline Total & $5,5 \pm 0,70$ & $14,0 \pm 1,38$ & $12,6 \pm 0,22$ \\
\hline
\end{tabular}

Médias não diferiram estatisticamente (Kruskal Wallis, $\mathrm{P}>0,05)$.

Tabela 3 - Médias ( \pm EP) do peso de frutos (g) e pupários (mg) e da área da asa direita de Anastrepha fraterculus (mm²) oriundas de goiabas (Psidium guajava) coletadas em Porto Alegre de fevereiro a março de 2009.

\begin{tabular}{lccc}
\hline \multirow{2}{*}{ Estágio de maturação/local } & \multicolumn{2}{c}{ Peso } & \multirow{2}{*}{ Área da asa $\left(\mathrm{mm}^{2}\right)$} \\
\cline { 2 - 3 } & frutos $(\mathrm{g})$ & pupários $(\mathrm{mg})$ & $12,8 \pm 0,26$ \\
\hline Verde & $61,1 \pm 5,04 \mathrm{~b}^{*}$ & $14,3 \pm 0,88$ & $13,0 \pm 0,38$ \\
Semimaduro & $92,8 \pm 9,38 \mathrm{a}$ & $17,7 \pm 0,74$ & $12,9 \pm 0,28$ \\
Maduro & $79,2 \pm 6,14 \mathrm{a}$ & $18,1 \pm 1,71$ & $12,9 \pm 0,18$ \\
Copa (total) & $77,4 \pm 4,34$ & $16,6 \pm 0,68$ & $13,3 \pm 0,26$ \\
Solo & $74,2 \pm 8,36$ & $16,5 \pm 1,28$ & $12,9 \pm 0,20$ \\
\hline Total & $76,5 \pm 3,85$ & $16,0 \pm 0,60$ & \\
\hline
\end{tabular}

* Médias seguidas de mesma letra minúscula nas colunas, não diferem significativamente (Tukey, P > 0,05). 
Tabela 4 - Médias ( \pm EP) do peso de frutos (g) e pupários (mg), e da área da asa direita de Anastrepha fraterculus $\left(\mathrm{mm}^{2}\right)$ oriundas de goiabas-serranas (Acca sellowiana) coletadas em Montenegro, RS, durante o mês de março de 2009.

\begin{tabular}{lccc}
\hline \multirow{2}{*}{ Estágio de maturação/local } & \multicolumn{2}{c}{ Peso } & \multirow{2}{*}{ Área da asa $\left(\mathrm{mm}^{2}\right)$} \\
\cline { 2 - 3 } & frutos $(\mathrm{g})$ & pupários $(\mathrm{mg})$ & \\
\hline Copa & $14,2 \pm 1,52 \mathrm{a}^{*}$ & $10,8 \pm 0,81$ & $12,3 \pm 0,37$ \\
Solo & $17,6 \pm 1,13 \mathrm{~b}$ & $12,1 \pm 0,85$ & $12,1 \pm 0,44$ \\
\hline Total & $15,9 \pm 0,97$ & $11,5 \pm 0,59$ & $12,2 \pm 0,28$ \\
\hline *Médias seguidas de mesma letra minúscula nas colunas não diferem significativamente (Kruskal Wallis, P $>0,05)$.
\end{tabular}

\section{DISCUSSÃO}

Com base no peso dos pupários, a goiaba e o araçá-vermelho parecem ter fornecido as condições mais satisfatórias para o desenvolvimento nas fases imaturas de $A$. fraterculus. O alimento, conforme PANIZZi; PARRA (1991), constitui a condição básica para a formação o desenvolvimento dos insetos. Segundo os autores, todos os processos ecológicos, fisiológicos e comportamentais ocorrem em um contexto nutricional. No caso de insetos carpófagos, como as moscas-das-frutas, de frutos maiores e com melhor composição nutricional, são esperados adultos com melhor desempenho, especialmente no tocante ao sucesso reprodutivo, o qual está relacionado à ingestão, durante a fase larval, de dois nutrientes primários, proteínas e carboidratos (ZuCOLOTO, 2000).

Duas características da fase larval são importantes para que um inseto adulto seja reprodutivamente competitivo: o tamanho, que influencia no sucesso da cópula, e o peso, que indica a condição nutricional armazenada eque afetará diretamente a fecundidade (PANIZZI; PARRA, 1991). Na fase imatura a quantidadee a qualidade dos nutrientes ingeridos afetam o peso, $\mathrm{o}$ tempo de desenvolvimento, a composição química do corpo, o tamanho do adulto e a produção de óvulos (Zucoloto, 2000). Segundo Panizzi; PARRa (2009), os insetos necessitam uma série de substâncias para o desenvolvimento, tais como carboidratos, lipídios, proteínas, vitaminas e sais minerais.

O teor de proteína da goiaba variedade Paluma referido por Gouveia et al. (2004) varia entre 0,74 a $0,82 \%$, no araçá-vermelho este teor é bem mais alto, $3,9 \%$ (GALHO et al., 2007) e a goiaba-serrana tem o menor valor 0,11\% (KINUPP; BARROS, 2008). A baixa concentração de proteínas pode, no caso da goiabaserrana, estar interferindo no peso dos pupários, uma vez que os oriundos destes frutos foram os mais leves. Esta suposição se apóia no trabalho de PANIzZI; PARRA (2009) que referem que as proteínas são, dentre os nutrientes, as que mais influenciam no desenvolvimento do inseto, pois estão diretamente relacionadas com a construção e a manutenção dos tecidos, interferindo na formação de estruturas de sustentação, assim como de órgãos. A concentração de proteínas pode acarretar em algumas vantagens em relação ao desenvolvimento do adulto, principalmente sob o aspecto reprodutivo (ZuColoto, 2000).

Em araçá-vermelho, observou-se que a correlação entre peso de pupários e o de frutos ocorreu quando estes estavam no estágio maduro. Nesta mesma espécie frutífera, em frutos coletados no solo, $20 \%$ da variação do peso dos pupários é explicada em função do peso dos frutos. Resultados que também foram observados em relação ao peso de frutos e de pupários, para frutos coletados no solo em goiaba-serrana (19\%). CRUZ et al. (2000) ressaltam a preferência alimentar de larvas de mosca-das-frutas por frutos hospedeiros em estágio maduro, pelo efeito fagoestimulante devidoà riqueza em açúcares. Fatores como a cor, textura e voláteis emitidos pela planta também interferem na escolha do fruto pela mosca para a oviposição. O sucesso reprodutivo das moscas-das-frutas depende da habilidade da fêmea em encontrar um hospedeiro que forneça todos os nutrientes para o imaturo (ZUCOLOTO, 2000). Outro aspecto a se considerar é o de que as necessidades nutricionais dos insetos não são constantes, variando de acordo com fatores bióticos (fases de desenvolvimento) e abióticos (temperatura, umidade etc) (PANIZZI; PARRa, 2009). Portanto, a disponibilidade do recurso alimentar, bem como sua qualidade, quando disponíveis, são fatores que acabam determinando o pesoetamanho dosindivíduose, consequentemente, o sucesso reprodutivo.

As goiabas, no presente estudo, foram, em média, os frutos mais pesados. As correlações negativas registradas entre peso de frutos e o de pupários para frutos verdes, maduros e para o total da planta, sugerem que o tamanho do fruto não foi oúnico fator que afetou o desenvolvimento das moscas-das-frutas. $\mathrm{O}$ levantamento de dados deinfestação também poderá contribuir para avaliar a competição por alimento que, segundo Souza (2007), também pode exercer influência sobre características morfométricas dos indivíduos. Frutos maiores como a goiaba e a goiabaserrana proporcionam recurso para abrigar um maior número de indivíduos por fruto, entretanto, há um aumento da competição por alimento, o que pode ocasionar uma diminuição do peso e do tamanho dos indivíduos. Segundo LomônACO; GERMANOs (2001), parece haver consenso de que a densidade constitui 
um fator causador de estresse, não somente porque promove a competição exploratória por recursos, mas também porque causa alterações químicas no substrato alimentar decorrentes do metabolismo larval.

Os resultados do presente trabalho indicam que, nestas espécies de frutíferas, o peso de frutos, onde as larvas de moscas se desenvolveram, influenciou, sob algumas condições, o peso de pupários. Entretanto, as relações entre peso de pupários e tamanho deasas, embora tenham ocorrido em alguns casos, mostrou correlações fracas conforme CALLEGARI-JACQUES (2003). Segundo a autora, avaliando qualitativamente o grau de correlação entre dois fatores o valor de $r$, ou seja, o coeficiente de correlação, determina que os eles variam entre regular ( $\mathrm{r}$ entre 0,3 a 0,6 ) e fraco (r entre 0 a 0,3) (Tabelas 2,4, 6 e 8).

Opeso dos frutos ea área das asas de A. fraterculus, em todas as espécies de mirtáceas, não evidenciaram correlação. Estes dados podem ser relacionados ao fato da espécie ter uma gama grande de espécies de frutíferas como hospedeiras, 76 conforme ZuccHI (2008). A adaptação a diferentes fontes de alimento no que se refere às espécies frutíferas utilizadas pelas larvas da mosca pode lhes garantir vantagens em relação à disponibilidade de alimento durante todo o ano ou pelo menos parte dele. O fato das larvas da mosca-das-frutas se desenvolverem de forma regular em mais do que uma espécie de mirtácea, garante recursos alimentares durante um longo período de tempo, que é a fase de frutificação das diferentes espécies frutíferas, possibilitando o desenvolvimento e a reprodução ao longo de todo ano.

\section{REFERÊNCIAS}

CALLEGARI-JACQUES, SIDIA M. Bioestatística princípios e aplicações. Porto Alegre: Artmed, 2003. 255p.

CARVALHO, R.S.; NASCIMENTO, A.S.; MATRANGALO, W.J.R. Controle Biológico. In: MALAVASI, A.; ZUCCHI, R. A. (Ed.). Moscas-das-frutas de importância econômica no Brasil: conhecimento básico e aplicado. Ribeirão Preto: Holos, 2000. p.113-117.

CLARO, S. A. Referenciais tecnológicos para a agricultura familiar ecológica: a experiência da Região Centro-Serra do Rio Grande do Sul. Porto Alegre: Emater/RS-ASCAR, 2001. 250p.

CRUZ, I.B.M. da; NASCIMENTO, J.C. do; TAUFER, M.; OLIVEIRA, A.K. de Morfologia do aparelho reprodutor e biologia do desenvolvimento. In: MALAVASI, A.; ZUCCHI, R.A. (Ed.). Moscas-das-frutas de importância econômica no Brasil: conhecimento básico e aplicado. Ribeirão Preto: Holos, 2000. p.55-66.

GALHO, A.S.; LOPES, N.F.; BACARIN, M.A.; LIMA, M. da G. de S. Composição química e respiração de crescimento em frutos de Psidium Cattleyanum Sabine durante o ciclo de desenvolvimento. Revista Brasileira de Fruticultura, v.29, n.1, p.61-66, 2007.

GOUVEIA, J.P.G. de; ALMEIDA, F. de A.C.; MEDEIROS, B.G. de S.; RIBEIRO, C.A. de F.A.; DUARTE, S.M.A. Determinação de características físico-químicas da goiaba: goiabeiras adubadas no Semi-Árido da Paraíba. Revista Brasileira de Produtos Agroindustriais, v.6, n.1, p.35-38, 2004.

GRELLMANN, E.O.; LOECK, A.E.; SALLES, L.A.B.; FACHINELLO, J.C. Necessidades térmicas e estimativa do número de gerações de Grapholita molesta (Busck, 1916) em Pelotas, RS. Pesquisa Agropecuária Brasileira, v.27, n.7, p.999-1004, 1992.

JOÃO, P.L. Levantamento da fruticultura comercial do Rio Grande do Sul. Porto Alegre: EMATER/RS - ASCAR, 2004. 89p.

KINUPP, V.F.; BARROS, I.B.I. de Teores de proteína e minerais de espécies nativas, potenciais hortaliças e frutas. Ciência e Tecnologia de Alimentos, v.28, n.4, p.846-857, 2008

KOVALESKI, A; SUGAYAMA, R.L.; URAMOTO, K.; MALAVASI, A. Rio Grande do Sul. In: MALAVASI, A.; ZUCCHI, R.A. (Ed.). Moscas-das-frutas de importância econômica no Brasil: conhecimento básico e aplicado. Ribeirão Preto: Holos, 2000. p.285-290.

LOMÔNACO, C.; GERMANOS, E. Variações fenotípicas em Musca domestica L. (Diptera: Muscidae) em resposta à competição larval por alimento. Neotropical Entomology, v.30, n.2, p.223-231, 2001.

MALAVASI, A.; ZUCCHI, R.A.; SUGAYAMA, R.L. Biogeografia. In: MALAVASI, A.; ZUCCHI, R.A. (Ed.). Moscas-das-frutas de importância econômica no Brasil: conhecimento básico e aplicado. Ribeirão Preto: Holos, 2000. p.93-98.

PANIZZI, A.R.; PARRA, J.R.P. Ecologia nutricional de insetos e suas implicações no manejo de pragas. São Paulo: Manole, 1991.350p.

PANIZZI, A.R.; PARRA, J.R.P. Bioecologia e nutrição de insetos: base para o manejo integrado de pragas. Brasilia: Embrapa Informação Tecnológica, 2009. 1164p.

PIRES, E.M. Estágios imaturos, morfologia interna e morfometria de Plastyscytus decempunctatus (Carvalho, 1945) (Heteroptera: Miridae). 2006. 72f. Dissertação (Mestrado em Entomologia) - Universidade Federal de Viçosa, Viçosa, 2006.

SILVA, T.G.A. Caracterização morfométrica e molecular de Anastrepha bistrigata Bezzi e Anastrepha striata Schiner (Diptera: Tephritidae). 2008. 65f. Dissertação (Mestrado em Ciências - Entomologia), Escola Superior de Agricultura Luiz de Queiroz, Universidade de São Paulo, Piracicaba, 2008. 
SOUZA, S.A.A. et al. Índices de infestação de Spondias lutea L. por moscas-das-frutas (Diptera: Tephritdae) e seus parasitóides no município de Seropédica, RJ. Magistra, v.29, n.1, p.25-30, 2007.

VAZ, L.A.L.; TAVARES, M.T.; LOMÔNACO, C.

Diversidade e tamanho de himenópteros parasitóides de Brevicoryne brassicae L. E Aphis nerii Boyer de Fonscolombre (Hemiptera: Aphididae). Neotropical Entomology, v.32, n.2, p.225-230, 2004.

ZUCCHI, R.A. Espécies de Anastrepha, sinonímias, plantas hospedeiras e parasitóides. In: MALAVASI, A.; ZUCCHI, R.A. (Ed.). Moscas-das-frutas de importância econômica no Brasil: conhecimento básico e aplicado. Ribeirão Preto: Holos, 2000a. p.41-48.

ZUCCHI, R.A. Taxonomia. In: MALAVASI, A.; ZUCCHI, R.A. (Ed.). Moscas-das-frutas de importância econô- mica no Brasil: conhecimento básico e aplicado. Ribeirão Preto: Holos, 2000b. p.13-24.

ZUCCHI, R. A. Fruit flies in Brazil- Anastrepha species and their hosts plants. 2008. Disponivel em: $<$ www.lea.esalq. usp.br/anastrepha/>.Acesso em: 17 abr. 2010.

ZUCOLOTO, F.S. Alimentação e nutrição de Moscadas-frutas. In: MALAVASI, A.; ZUCCHI, R.A. (Ed.). Moscas-das-frutas de importância econômica no Brasil: conhecimento básico e aplicado. Ribeirão Preto: Holos, 2000. p.67-80.

Recebido em 26/4/10

Aceito em 7/1/11 